\title{
Bootstrapping a Location Service Through Geocoded Postal Addresses
}

\author{
Gayathri Chandrasekaran, Mesut Ali Ergin, Marco Gruteser, \\ and Richard P. Martin \\ WINLAB, Electrical and Computer Engineering Department \\ Rutgers, The State University of New Jersey \\ North Brunswick, NJ 08902-3390 \\ $\{$ chandrga, ergin, gruteser\}@winlab.rutgers.edu, rmartin@cs.rutgers.edu
}

\begin{abstract}
We analyze the feasibility of boostrapping a location service through geocoded postal addresses rather than the common wardriving technique. A location service that contains the MAC addresses and geographic position of wireless LAN access points enables positioning services for WLAN devices and location-aware networking protocols. This work thus compares the accuracy of access point position estimates obtained based on RF signal strengths readings (wardriving) with the accuracy of the geocoded postal address. The results show similar accuracy for geocoding in comparison to typical wardriving studies, with significantly reduced effort if postal addresses of access point positions are known.
\end{abstract}

\section{Introduction}

WiFi localization promises to complement satellite positioning in two key areas that frequently experience poor satellite coverage, indoor environments and urban canyons. Wide area WiFi localization techniques as provided by Place Lab [1] or commercial providers [2], however, rely on a location service that provides geographic position of third-party WiFi access points identified by their MAC address.

Obtaining the data to initialize and subsequently maintain the records of such a location service requires significant effort. Typically, data is collected via wardriving, whereby a vehicle sweeps the area of interest and recording periodically its position and the signal strength of nearby access points (the vehicle can determine its position with a Global Positioning System receiver). From this dataset, the position of access points can then be estimated using a number of heuristics such as position of maximum signal strength, or centroid of all access point sightings 1 This process is inaccurate 4 and it is laborious, since it requires physically driving a vehicle through the road network in the intended coverage area. It needs to be repeated periodically to update the dataset, since owners of access points upgrade their hardware (resulting in a change of MAC address),

${ }^{1}$ See Wigle.net 3] for example wardriving datasets. 
or move with the access point to a different geographic location. To reduce this updating effort [5] proposes self-mapping, wherein users of the location service contribute updates to its records. Still, bootstrapping a location service to the point where it is useful for early adopters is necessary.

This paper studies the feasibility of an alternate method, initializing location service records through geocoded postal addresses. This approach is motivated by the realization that large Internet service providers already maintain a directory of postal addresses (i.e., billing addresses) and could easily add MAC addresses to these records. To evaluate this approach, we compare the accuracy of access point position estimates obtained through wardriving with those from postal address geocoding. Specifically, we study the effect of building and road densities, wardriving road coverage, and geocoding databases on these results.

The remainder of this paper is organized as follows. The terms and metrics are introduced in section 2. The methodology is described in detail in section 3. Section 4 describes the results and section 5 has discussions related the ideas presented. The related work is summarized in Section 6. Finally, section 7 concludes the paper.

\section{Terms and Metrics}

In this section, we define the various terms and metrics used in the paper. Definitions are as follows:

- Actual Location of the AP: It is the geographic location where the AP is physically installed. This was found using the Orthophoto-based geocoding explained in the next section.

- Full Coverage War-Driving Location of the AP: It is the geographic location of the AP reported by war driving through both the main and side roads. The inference of the location of the AP in this experiment is based on the location at which the strongest SNR was received. Full Coverage War Driving Error is then defined as the Euclidean distance between the actual location of the AP and the full coverage war driving location of the AP, measured in feet.

- Main Road War-Driving Location of the AP: It is the geographic location of the AP reported by war driving only through the main roads. Main Road War-Driving Error is then defined as the Euclidean distance between the actual location of the AP and the main road war driving location of the AP, measured in feet.

- Postal Address Geocoded Location of the AP: It is the geographic location of the AP reported by geocoding the postal address of the building in which the AP is installed. Postal Address Geocoding Error is then defined as the Euclidean distance between the actual location of the AP and the postal address geocoded location of the AP, measured in feet. 


\section{Methodology}

In this section, we define our goals and explain our experimental techniques used to achieve these goals. Our goals were to:

1. Quantify the errors due to different kinds of war driving efforts. In our experiments, we compare two different kinds of war-driving - Full Coverage War Driving which involves driving through both main roads and side roads surrounding the area of interest to get a comprehensive coverage of the APs, and the Main Road War-Driving, which is typically similar to the kind one can find in the public war driving databases, where only the major roads around the area of interest are traversed.

2. Analyze the postal address geocoding error for two different map data sources, namely the Navteq and TIGER/Line. Navteq [6] serves as the main data source for Yahoo Maps, Google Maps, and Mapquest. TIGER/Line [7] map data source is being developed by the US Census Bureau for supporting imaging needs of the Decennial Census and other bureau programs.

3. Quantify the errors incurred with various bootstrapping techniques in three different environments: A sparse residential area (Figure 1), a relatively dense residential area (Figure 2), and a highway (Figure 3). The sparse residential area consisted of single family houses which had long walkways between the roads and the houses. The relatively dense Residential area had single family houses closer to the roads. Highway had three lanes on each direction with $80 \mathrm{~km} / \mathrm{h}$ speed limit and mostly consisted of commercial buildings. All areas were within central New Jersey region.

4. Study our claim that postal address geocoding techniques could complement or partially replace the war driving efforts while still giving comparable results in terms of accuracy.

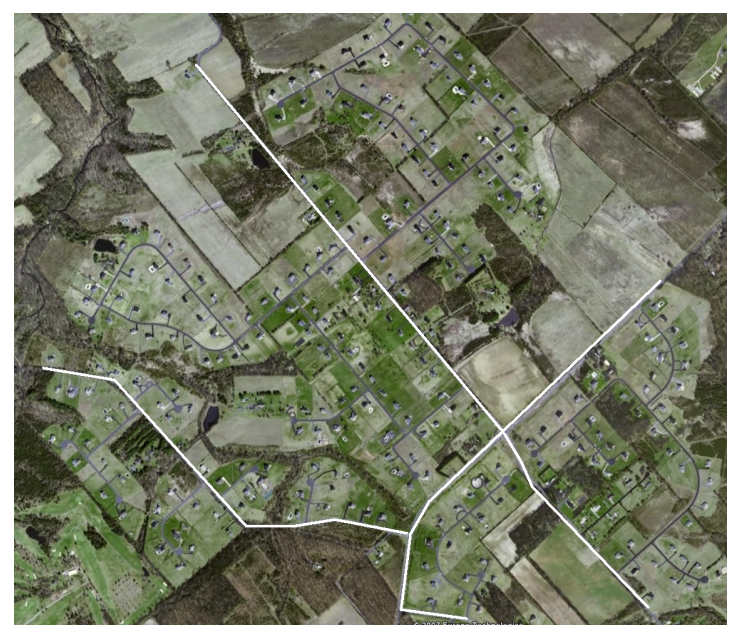

Fig. 1. Sparse Residential Area. The white line represents the route driven for Main Road War Driving. 


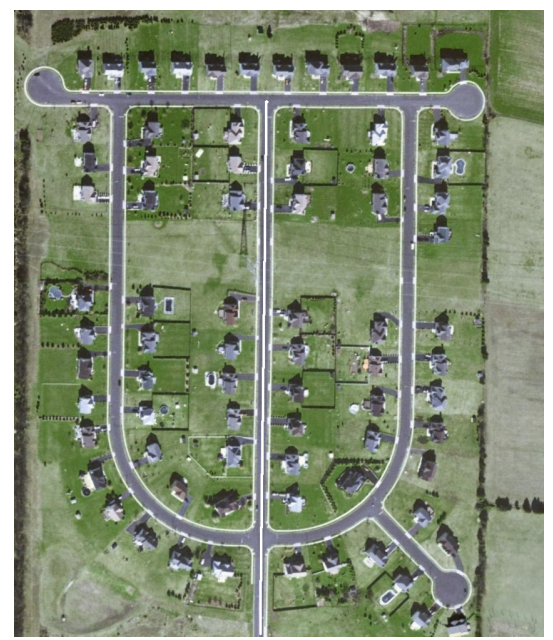

Fig. 2. Relatively Dense Residential Area. The white line represents the route driven for Main Road War Driving.

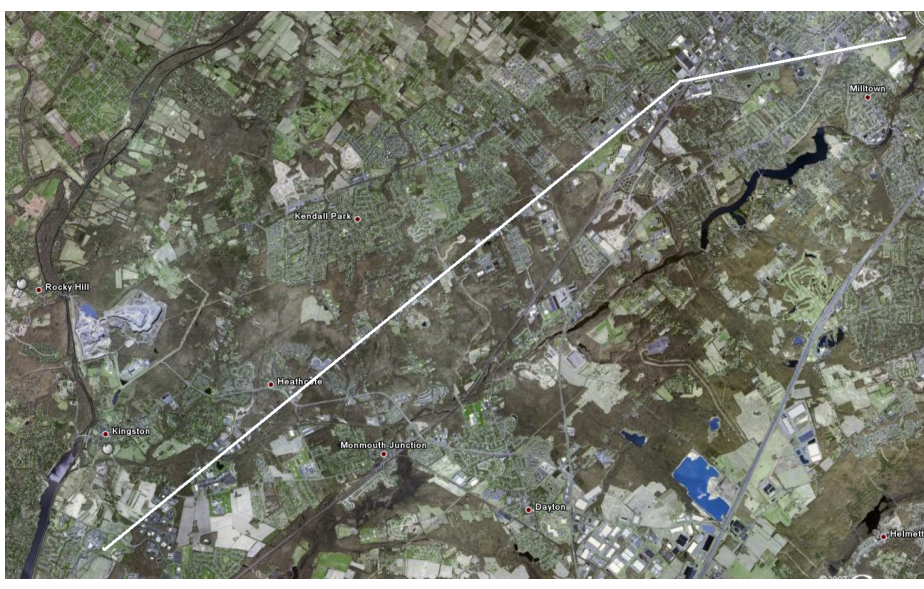

Fig. 3. Highway Route. The white line represents the route driven in the highway.

Towards achieving stated goals, we first need to know the actual postal addresses where the access points are installed in our selected areas. Our final dataset consisted of all these APs for which we were able to determine the corresponding postal address of their installation location. The total number of such APs are 167 in this paper. For each of these APs, orthophoto-based geocoding was accomplished, where we visually identified each of these postal address on Google Earth [8] and geocoded the address to a location centered on the building related to the address. Then, the corresponding errors for the different 


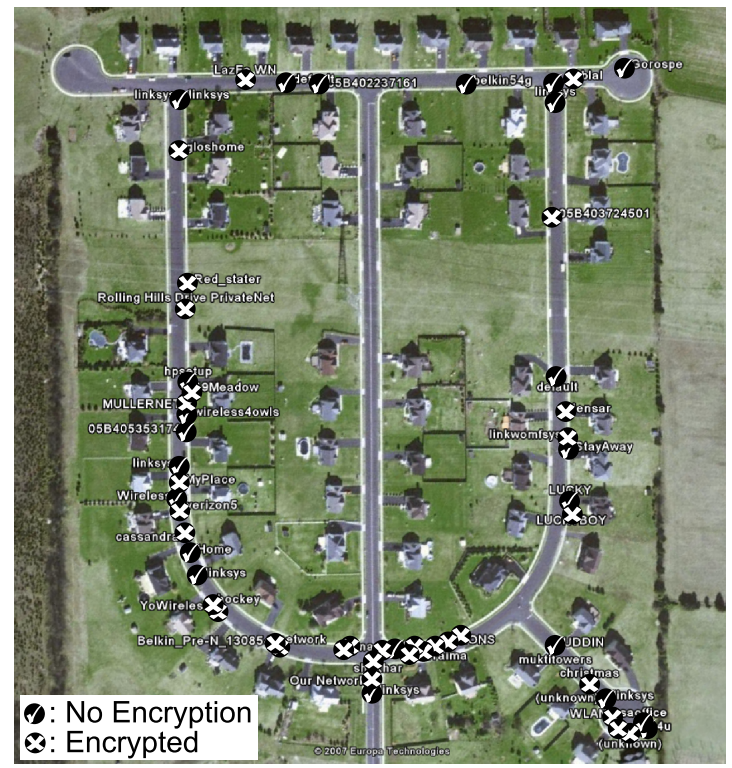

Fig. 4. The positions of the APs reported from war driving with a directional antenna

bootstrapping techniques were determined by comparing them to the data obtained in this step. We summarize the steps involved in this process as:

1. Locate the access points and the postal address of the place where the APs were deployed with the help of a directional antenna.

2. Create an orthophoto-based geocoding for each of the postal addresses that we determined in the first step to establish the ground truth about the actual geographic location of the AP.

3. War drive on main and small roads with an omni-directional antenna to obtain a comprehensive coverage of the region and determine the geographic locations of the APs as reported by war driving.

4. War Drive only on main roads with an omnidirectional antenna and determine the geographic locations of the APs as reported by war driving.

5. Geocode all the postal addresses collected in Step 1 using the Navteq and the TIGER/Line databases.

\subsection{Actual Location of the AP}

Identifying the postal address where the APs were deployed was challenging due to the dense deployment of the $802.11 \mathrm{a} / \mathrm{b} / \mathrm{g}$ Access points.

Figure 4 illustrates the war driving data obtained with a typical omnidirectional antenna in the relatively dense residential area. We can see from the figure that it is difficult to determine in which buildings the access points are placed.

Hence, in addition to the external USB-driven Pharos iGPS-360 GPS unit, we decided to use a $2.4 \mathrm{GHz}, 15 \mathrm{dBi}$, Die Cast Mini-Reflector Grid Wireless LAN 
directional antenna for war driving. The directional antenna was connected to a Hermes chipset based Orinoco Silver PCMCIA wireless card in the laptop using an N-Male to MC converter. We pointed our directional antenna towards the outer edge of the circular area. The sniffed frames were fed into the stumbling software (Network Stumbler [9]) and the locations of the APs were plotted. The new plot looked similar to 4 but the SNR readings in each of the plotted APs gave an insight about the direction from which the signals were captured.

We could see that each AP plotted on the linear-segment of the road in Figure 4 could correspond to one of the two houses on either sides of the road (because we are plotting APs on points where the best signal strength was received). We then looked at the SNR of the each of these APs to determine whether the AP signal came from the direction in which the directional antenna was pointed to or from the other side. According to the specification, the antenna has a differential gain between front and back lobes of $20 \mathrm{~dB}$ and a horizontal beamwidth of 16 degrees. Therefore, we were able to observe peak RSSI values above 80 when the antenna pointed directly at the home containing the access point, and RSSI values dropped by about 10 points when the antenna faced backwards. We determined these thresholds through a calibration procedure on several freestanding houses (where we were certain about the AP position). Based on this calibration, we decided to map an AP onto the house facing the antenna, if its maximum RSSI values lies above 80, map it onto the house in the opposite direction if its value lies between 75 and 80, and drop all other points to reduce mapping uncertainty. In the dense area, we also verified a sample of 3 access point locations with residents of the community, all of which were correctly mapped by this procedure.

The highway mostly had commercial buildings like hotels, restaurants, coffeeshops, book-stores, whose SSIDs were more meaningful and matched with the commercial spot's name. Table 1 summarizes a few of our observations of the common SSIDs used by these commercial spots.

Since multiple APs were installed in hotels with the same SSIDs, it was usually simple to identify the correspondence between the AP name and the location at which it was installed by looking at the war driving data.

Thus for the highway area, we drove once with an Omni-Directional Antenna to capture the wireless signals and extracted the SSIDs that we were able to

Table 1. Correlation between the enterprise trademark names of the commercial buildings and their Wireless SSIDs

\begin{tabular}{|c|c|}
\hline Commercial Name & SSID \\
\hline Barnes and Nobles & BNDEMO \\
\hline Holiday Inn & HOLIDAY INN \\
\hline Best Western & ethostream \\
\hline Extended Stay America & goesh \\
\hline Panera Bread & PANERA \\
\hline Red Roof Inn & tmobile \\
\hline Midas Auto Services & MIDASWIRELESS \\
\hline
\end{tabular}




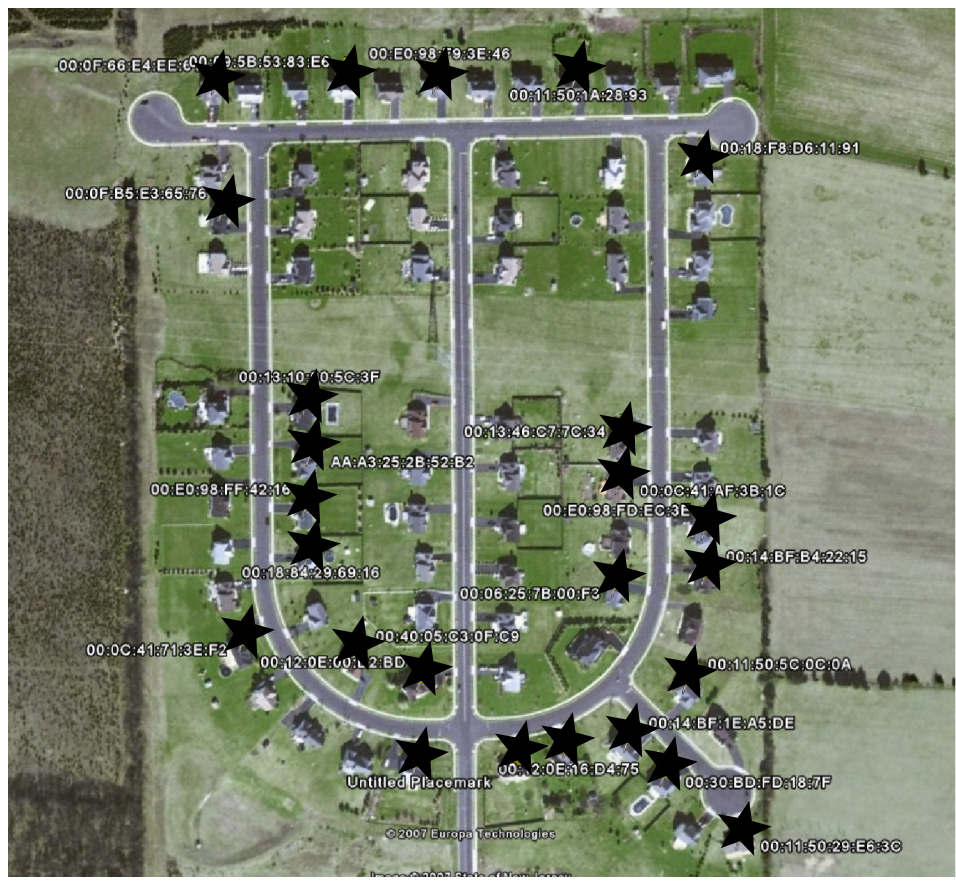

Fig. 5. Orthophoto-based geocoding for the postal addresses along with the MAC address of the AP installed in that address

correlate with the commercial spot. We then drove once again to note down the postal addresses of the commercial spots that we identified in the first run. Thus the ground truth of the location/postal address of the APs in the highway was established.

\subsection{Orthophoto-Based Geocoding}

For each of the postal addresses collected in the previous step, we did an orthophoto-based geocoding. In this process, we visually identified each of these postal address on Google Earth [8] and geocoded the address to a location centered on the building referenced by the address. Thus for each of the postal addresses, we extracted the actual geocodes.

Figure 5 represents the orthophoto-based geocoded postal addresses along with the MAC address of the AP installed at that particular postal address. Each star in Figure [5 is placed on the center of the house and is labeled with the MAC address of the AP installed in that house.

\subsection{War Driving}

War driving is widely used to estimate access point locations. War driving is the process of gathering statistics about wireless networks in a given area by 
listening to the broadcast beacons emitted by the APs. These beacons contain information regarding the SSID, MAC, and encryption capabilities. Along with a GPS unit, these beacons can be used to log available networks using various freely available war driving software such as NetStumbler [9] or Kismet [10]. We used Network Stumbler to collect our logs.

There are various algorithms to infer the actual location of the AP from war driving logs. Centroid, weighted-centroid, and particle filters 11 are examples to those algorithms used in tools like [12,13. However, since most paths that we drove were approximately linear, the approximated location of the APs fell on this linear segment. Hence, we used the point at which maximum signal to noise ratio was achieved as an estimate of the location of the AP in our war driving data.

Full Coverage War-Driving. For the full coverage war driving data, we drove on all the main roads and small roads at speeds less than $45 \mathrm{~km} / \mathrm{h}$. As explained before, the points of best signal strength for each of the APs were used and the Euclidean distance between the location of the AP reported by the full coverage war driving and the actual location of the AP (found using step 2) was calculated.

Main Road War-Driving. Public war driving repository like Wigle.net 3] allow users of the community to upload their war driving data, containing information about the BSSID and locations of the APs encountered. The serious drawback of this data is that the APs are primarily located near the main roads and commercial areas due to the community members' driving patterns. It is impractical to achieve the accuracy of full coverage war driving by using the data from these repositories. Therefore, in our evaluations of the residential areas, we considered driving only on main roads (as shown by the white lines in Figures 1 and 2) and quantified the Euclidean distance between the AP location reported by main road war driving and the actual location of the AP.

\subsection{Geocoding Postal Addresses}

In general, the postal address geocoding is accomplished by

- Geocoding the end points of the road segments

- Noting down the address range in each of these segments

- Extrapolating the geographic co-ordinates for the remaining addresses in that segment.

Thus there is some error introduced due to the extrapolation step. If the road segments are long and if the houses/commercial buildings are not equispaced, the error introduced due to extrapolation of the geographic co-ordinates of the addresses would be high. Similarly, small road segments introduce less extrapolation error. In our experiment, the US1 highway roads had long road segments whereas the residential areas had shorter road segments.

In our evaluations, we have considered two different Address Map Databases Navteq [6] and TIGER/Line [7] for the characterization of the accuracy of the 
postal address geocoding. Navteq serves as the data source for Yahoo Maps, Google Maps, Google Earth and MapQuest. So, we evaluate only the geocodes for the postal address generated by the Yahoo Maps among the other alternatives that use the same data source.

The TIGER/Line system (Topologically Integrated Geographic Encoding and Referencing) is a different digital database developed at the U.S Census Bureau to support its imaging needs for the Decennial Census and other Bureau programs. The median accuracy of this data is claimed to be around $167 \mathrm{ft}$. Thus, we also evaluate the geocoding accuracy of this database for the postal addresses that have been generated.

We report the postal address geocoding error for the mentioned two address map databases in our evaluations.

\section{Results}

Figure 6] shows the error distributions for the sparse and the relatively dense residential areas. The sparse residential area that we considered had single family houses that were far from the roads with huge walkways. Thus the full coverage war driving results in Figure 6(a) show a median error of around 300ft. The relatively dense residential area had single family houses close to the roads. Hence the median error for the full coverage war driving in relatively dense residential area is about $110 \mathrm{ft}$, as shown in 6(b). Similar patterns in the sparse and dense residential areas are observed for the main road war driving where the median error for sparse residential area $(\sim 1000 \mathrm{ft})$ is much higher than median error for the relatively dense residential area $(\sim 500 \mathrm{ft})$.

The error due to postal address geocoding is not very much affected by the type of region. Median error exhibited by both the Yahoo Maps and TIGER/Line database in either case is around $300 \mathrm{ft}$ to $350 \mathrm{ft}$. However, we observe from

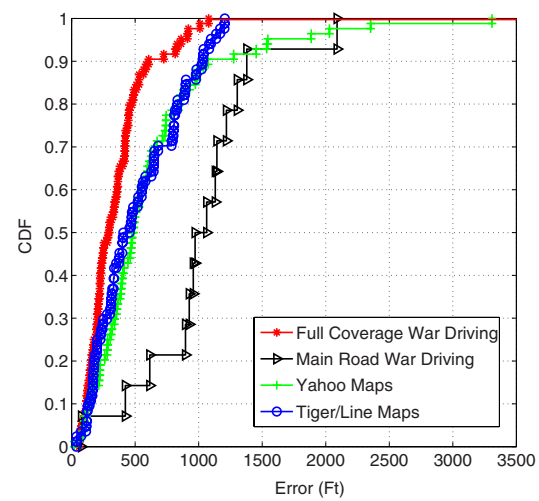

(a)

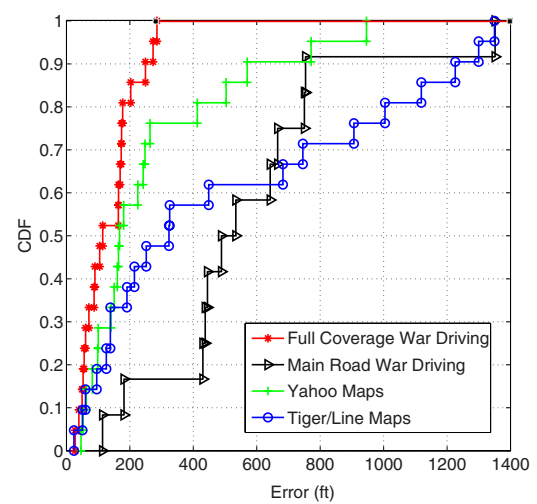

(b)

Fig. 6. (a) Error distribution in sparse residential area, (b) Error distribution in relatively dense residential Area 
Figure 6(b) that Yahoo Maps have a much better geocoding compared to the TIGER/Line maps. The median error for Yahoo maps in the case of relatively dense residential area is around 180ft as opposed to TIGER/Line database, which is around $300 \mathrm{ft}$.

In Figure 7, we only plot one type of war driving result (i.e., main road war driving), since highway road is indeed considered to be the only road surrounding the area. In other words, there is no difference between the full coverage war driving and main road war driving for the highway area. Obviously, we could have driven in the parking lots to improve the accuracy, however, this is beyond the scope of our experiment and also considered trespassing for some of the buildings. We also do not show the error distribution of the TIGER/Line database in this figure, because the US-1 Highway addresses that we have considered in this experiment do not yet appear in the TIGER/Line Maps.

Our observations from the experiment highlight the fact that the accuracy of the highway postal address geocoding is low (with median errors of $750 \mathrm{ft}$ ). These high errors could be attributed to the long road segments in highways as discussed in the previous section. Although some of the popular highways have been subject to war driving, many others can still benefit from postal addressbased bootstrapping. Also, data from already covered highways can be refined further through the postal address-based approach, since quality of the map databases are continuously being improved for many other purposes.

Figure 8 summarizes the data for all three areas we had considered. Our overall observations follows:

- As expected the time-consuming full coverage war driving method provides the least AP position estimation error, a median error of $250 \mathrm{ft}$.

- Main road war driving, which is less time consuming than full coverage war driving, reduces accuracy to a median of $350 \mathrm{ft}$.

- Both Yahoo Maps and the TIGER/Line maps exhibit similar error characteristics for most addresses. However, Yahoo geocoding shows more serious outliers with errors up to $3300 \mathrm{ft}$. In general, $95 \%$ of the geocoded postal addresses generated using either of the databases have errors less than $1200 \mathrm{ft}$.

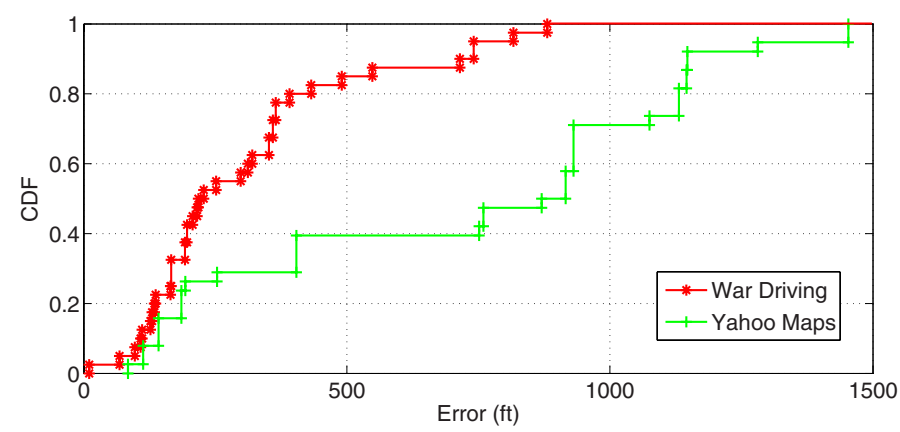

Fig. 7. Error Distribution in Highway Scenario 


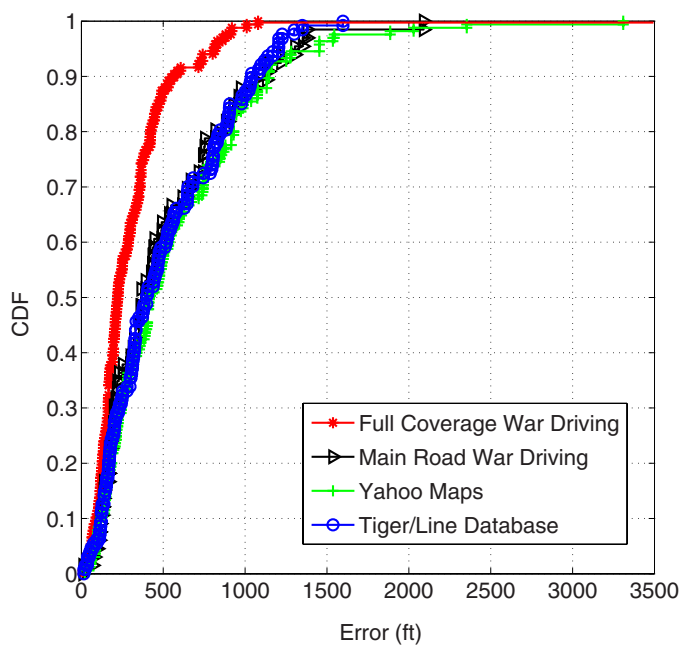

Fig. 8. Summary of the Error Distribution for Various Bootstrapping Procedures

- Postal address geocoding shows a median error of 350ft, similar to the accuracy of main road war driving technique in the areas that we have considered. (However, main road war driving can be less accurate if sufficient coverage is not achieved, as in the residential areas shown earlier).

Table 2 summarizes the number of APs detected through various bootstrapping procedures in the three areas we considered - Sparse residential area, relatively dense residential area and highway. In total, we analyzed a sample of 167 access points from all three areas. The bottleneck for extending this sample of access points was our inability to identify without ambiguity the ground truth about the postal addresses of many other locations APs were deployed. Of 167 APs, 106 APs were selected from the sparse residential area due to the ease of identification of the actual locations of the APs.

We observe that full coverage war driving has a detection rate of $100 \%$ in all three areas. However, this result is biased because the ground truth for the

Table 2. Number of APs detected in various bootstrapping procedures

\begin{tabular}{|c||c|c|c|c|c|}
\hline & $\begin{array}{c}\text { Full Coverage } \\
\text { War Driving }\end{array}$ & $\begin{array}{c}\text { Main Road } \\
\text { War Driving }\end{array}$ & Yahoo Maps & $\begin{array}{c}\text { TIGER/Line } \\
\text { Database }\end{array}$ & $\begin{array}{c}\text { Total APs } \\
\text { Analyzed }\end{array}$ \\
\hline \hline $\begin{array}{c}\text { Sparse } \\
\text { Residential Area }\end{array}$ & 106 & 14 & 106 & 106 & 106 \\
\hline $\begin{array}{c}\text { Relatively Dense } \\
\text { Residential Area }\end{array}$ & 21 & 12 & 21 & 21 & 21 \\
\hline $\begin{array}{c}\text { US Highway 1 } \\
\text { Area }\end{array}$ & 40 & - & 38 & 0 & 40 \\
\hline
\end{tabular}


Table 3. Time taken (in mins) for full coverage and main road war driving

\begin{tabular}{|c|c|c|}
\hline & \multicolumn{2}{|c|}{ Time taken for War Driving in Mins } \\
\hline & Full Coverage War Driving & Main Road War Driving \\
\hline Sparse Residential Area & 30 & 3.5 \\
\hline Dense Residential Area & 6 & 2 \\
\hline Highway & 45 & 45 \\
\hline
\end{tabular}

presence of APs was established with the help of war driving with a directional antenna. APs which were not detected in war driving were not included in the experiment. The significance of this table is that main road war driving detects a far lesser number of APs than the full coverage war driving in the residential areas. The number of APs detected in the sparse residential area is only 14 out of 106 APs that were considered in that area. The number of APs detected in the relatively dense residential area is 12 out of 21 . The very low AP detection rate in the sparse residential area is due to the presence of a large residential community located far from the main roads as shown in Figure 1. The relatively dense residential community, however, is small and we traversed a path along the center of the community as shown by the white line in Figure 2 . As a result, $57 \%$ of the APs have been detected in this area. The number for main road war driving in the highway case is not different than the full coverage war driving case. The table also shows that both Yahoo Maps and TIGER/Line database have the geocodes for all of the residential addresses we have used. For the US1 highway, TIGER/Line Map does not have geocodes for any of the 40 addresses, whereas Yahoo Maps have geocodes for the 38 addresses out of these 40 . The absence of TIGER/Line data for the US1 highway should not be generalized for other highways without further investigation.

Table 3 summarizes the time taken (in Mins) for full coverage war driving and main road war driving in both of the residential areas.

We see that full coverage war driving, which took 30 mins in sparse residential area, is about nine times more time consuming than the main road war driving, which took around 3.5 minutes. Similarly, the full coverage war driving in relatively dense residential area took thrice as much time as that of main road war driving in the same area. In highways, our route of 25 miles took nearly 45 minutes of war drive time.

\section{Discussion}

In this section, we elaborate on accuracy considerations and methods for obtaining databases with MAC and postal addresses.

A key issue to make the geocoding approach feasible is obtaining a database that maps (wireless interface) MAC addresses to the postal addresses of their deployment locations. We envision several ways of obtaining such a database. First, access point software configuration wizards could be programmed to ask the end 
user or the installation technician for the postal address of the deployment location and report both to a location service. Due to the nature of the wireless communications at public frequencies, access points already contain a software face for installing and efficiently operating an AP at a certain location already require manual intervention (e.g, configuring channel, SSID and encryption settings). This approach would require participation of access point manufacturers. Alternatively, this software could be integrated into any downloadable software (presumably providing a service of interest to users to encourage downloading). This software can query the MAC address of the associated access points from the local wireless interface, and ask the user for the postal address (with appropriate disclosure). In both approaches, some users may be unwilling or unable to provide the postal address information. Some may either deliberately or accidentally enter incorrect information, a source of inaccuracy that was outside the scope of our study.

A different approach to obtaining such a database, relies on Internet service providers to record the MAC addresses of provided access points and the postal addresses where the Internet service is provided. Many current ISP contracts include a free wireless access point and a significant number of users is likely taking advantage of such offers. Similarly, major online retailers could combine wireless MAC and shipping addresses of access points. Again, these approaches introduce inaccuracies not considered in this study, when access points are moved to and used at a different address than the original shipment address. (ISP may be able to detect this via a change in MAC address connected to their network, but retailers can not). However, this approach may at least provide enough data to seed (or bootstrap a location service) in certain areas whereby wardriving may still be needed in others.

To reduce privacy concerns of users, the location service database should not contain the real identities of users and should not contain the postal addresses, only the geographic coordinates. If users harbor further concerns about an AP linked to their home locations, perturbations of the access point position could be introduced, although this would reduce accuracy.

Note also that the accuracy of geocoding continues to improve. High accuracy of this process is essential for many vital services (e.g. emergency response, law enforcement, planning delivery routes, etc.). Therefore, parallel attempts from both government and private sector exist to increase the fidelity of geocoding. In particular, the E911 Phase-II requirement is a driving force for geocoding. While it mainly concerns the accuracy of cellular positioning systems (requirement of less than 50 to $300 \mathrm{~m}$ error 14 ), these coordinates usually must be translated into postal addresses with which emergency responders are more familiar. Thus, the accuracy of this translation process is also important and future location services can benefit from these continuing geocoding refinements.

The postal-address-based techniques are likely not very accurate in the enterprise domain, because a significant number of such APs are deployed within campus locations, where the whole campus is referenced by a single street address to the external world. However, wardriving is still unnecessary if installation 
location information from conventional enterprise asset tracking or network management systems can be provided to the location service. This would, of course, require participation of the campus network administrators.

Last but not least, the accuracy implications of access point location errors on a client positioning service are dependent on the positioning algorithms used and remained outside the scope of this paper. While some indoor positioning services such as RADAR [15] do not rely on actual AP positions, to our knowledge, widearea services such as Place Lab [1] would be affected by AP position errors.

\section{$6 \quad$ Related Work}

War driving has gained importance with the launch of the Place Lab 1] initiative for the outdoor localization. Projects like Wigle.net maintain user contributed datasets containing the SSID, MAC and locations of the APs discovered through war driving. War driving is time consuming and it is desirable to look at alternatives to complement war driving efforts for efficient bootstrapping.

Authors of [16] characterize the error in location inference when localization is done using AP locations discovered from war driving. They, however, do not propose any alternative solutions to war driving and their evaluations are limited to a single campus network.

In 17, authors study accuracy and efficiency of wardriving process using three different methods: on foot, on bicycle, in car. Experiments show the position estimation's strong dependence on the particular means used for wardriving (e.g., wardriving on bicycle allows up to three times more APs to be discovered compared to wardriving in car).

Initial studies on the probability distribution of errors incurred by geocoding residential address using the TIGER/Line [7] data source appeared in [18. The authors have also had access to the (non-public) E911 database through the Iowa county officials and have reported the median geocoding error to be $145 \mathrm{ft}$. This result motivated us to evaluate geocoding for bootstrapping a location service, it can, however, not be directly compared to the accuracy results from previous wardriving studies, since these results are very dependent on the locale. Our work provided a direct comparison of wardriving and geocoding accuracies in the same areas. There are also other attempts to evaluate geocoding accuracy for various domains of study, like public-health [19] and transportation 20].

Also, 4, analyzed the Skyhook Wireless War Driving Database and has briefly illustrated the arterial bias involved in inferring the AP's location through war driving. Comprehensive wardriving on surrounding roads has been pointed out as a means to reduce this bias.

\section{Conclusion}

In this paper we have considered geocoding of postal addresses as an alternative method to populate a location service with WiFi access point positions. Specifically, we compared the accuracy of AP position estimations obtained through 
geocoding with that of wardriving. We found that in the considered areas, estimation accuracy through geocoding matched that of wardriving arterial roads. Accuracy through wardriving can be improved, however, by covering a larger fraction of the road network.

Based on these results we believe that geocoding postal address is a viable alternative to populate location services. Even partial postal address datasets might reduce wardriving effort in a hybrid approach involving both geocoding and wardriving. We hope that organizations in possession of large numbers of addresses (for installed AP locations) can use these insights to bootstrap WiFi localization services.

\section{References}

1. LaMarca, A., Chawathe, Y., Consolvo, S., Hightower, J., Smith, I., Scott, J., Sohn, T., Howard, J., Hughes, J., Potter, F., Tabert, J., Powledge, P., Botriello, G., Schilit, B.: Place lab: Device positioning using radio beacons in the wild. In: Proceedings of the third International Conference on Pervasive Compiting Pervasive, May 2005, pp. 116-133. Munich, Germany (2005)

2. Skyhook Wireless, http://www.skyhookwireless.com/

3. Wigle.net, http://www.wigle.net/

4. Jones, K., Liu, L.: What where wi: An analysis of millions of wi-fi access points. Tech. Rep., Georgia Institute of Technology, Atlanta, GA, USA (2007)

5. LaMarca, A., Hightower, J., Smith, I., Consolvo, S.: Self-mapping in 802.11 location systems. In: Beigl, M., Intille, S.S., Rekimoto, J., Tokuda, H. (eds.) UbiComp 2005. LNCS, vol. 3660, pp. 87-104. Springer, Heidelberg (2005)

6. Navteq, The Map Database provider for Yahoo/Google/MapQuest, http:// www. navteq.com/

7. Tiger/Line - Topologically Integrated Geographic Encoding and Referencing - A digital map database developed at the U.S. Census Bureau, http://www. census . gov/geo/www/tiger/tigerua/uatgr2k.html

8. Google Inc., Google Earth Release 4.1 (2007), http://earth.google.com/

9. Network Stumbler, http://www.netstumbler.com

10. Kismet, http://www.kismetwireless.net/

11. Hightower, J., Borriello, G.: Particle filters for location estimation in ubiquitous computing: A case study. In: Davies, N., Mynatt, E.D., Siio, I. (eds.) UbiComp 2004. LNCS, vol. 3205, pp. 88-106. Springer, Heidelberg (2004)

12. Earth Stumbler, http://www.earthstumbler.net/

13. Kismet Earth, http://www.kismetearth.net/

14. Enhanced 911 - Wireless Services, (February 2006), http://www.fcc.gov/ 911/enhanced/

15. Bahl, P., Padmanabhan, V.N.: RADAR: An in-building user location and tracking system. In: Proceedings of IEEE Infocom, March 2000, vol. 2, pp. 775-784. IEEE Computer Society Press, Los Alamitos (2000)

16. Kim, M., Fielding, J.J., Kotz, D.: Risks of using AP locations discovered through war driving. In: Fishkin, K.P., Schiele, B., Nixon, P., Quigley, A. (eds.) PERVASIVE 2006. LNCS, vol. 3968, pp. 67-82. Springer, Heidelberg (2006)

17. Yoshida, H., Ito, S., Kawaguchi, N.: Evaluation of pre-acquisition methods for position estimation system using wireless LAN. In: Proceedings of the Third International Conference on Mobile Computing and Ubiquitous Networking (ICMU 2006), London, UK, October 2006, pp. 148-155 (2006) 
18. Zimmerman, D., Fang, X., Mazumdar, S., Rushton, G.: Modeling the probability distribution of positional errors incurred by residential address geocoding. International Journal of Health Geographics 6(1) (2007)

19. Kravets, N., Hadden, W.C.: The accuracy of address coding and the effects of coding errors. Health and Place (Elsevier) 13(1), 293-298 (2007)

20. Indech-Nelson, J., Zuehlke, K.K., Guensler, R.: A comparison of geocoding methodologies for transportation planning applications. In: Proceedings of 11th TRB National Transportation Planning Applications (May 2007) 